\title{
Implizite Eigenkapitalkosten und der Fehler in den Analystenprognosen in der Schweiz
}

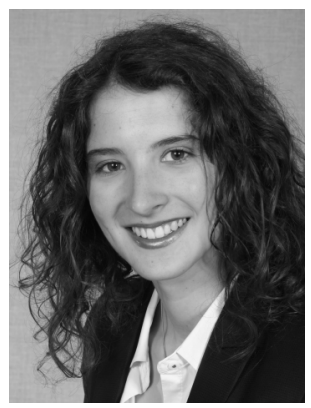

\section{Patricia Ruffing-Straube"}

Implizite Kapitalkosten, Unsicherheit, Markterwartungen, Analysten, Empirische Kapitalmarktforschung, Bewertung

Implied cost of capital, uncertainty, market expectations, analysts, empirical capital market research, valuation

In diesem Beitrag werden die impliziten Kapitalkosten für den Schweizer Aktienmarkt auf monatlicher Basis für einen Zeitraum von Januar 2006 bis Juni 2014 ermittelt. Diese stellen auf die aktuellen Erwartungen der Marktteilnehmer für die Zukunft ab und führen so zu zukunftsorientierten Werten für die Eigenkapitalkosten. Entsprechend den gestiegenen Unsicherheiten am Kapitalmarkt verzeichnet die Schweizer Marktrisikoprämie in der Finanzkrise von 2008 und im Sommer 2011 mit der zunehmenden Frankenstärke einen deutlichen Anstieg. Die Branchenrisikoprämien schwanken ebenfalls mit den konjunkturellen Veränderungen. Die Ergebnisse zeigen ausserdem, dass Analystenschätzungen für Schweizer Unternehmen auch in volatilen Zeiten verzerrt und ungenau sind, wobei Argumente für und gegen eine daraus resultierende Verzerrung der Kapitalkosten diskutiert werden.

In this paper, I derive the implied cost of capital for the Swiss capital market on a monthly basis for the period from January 2006 to June 2014. Implied cost of capital models consider the actual expectations of market participants for future periods when deriving the cost of capital. In line with increased uncertainty in capital markets, the Swiss market risk premium shows a substantial increase during the financial crisis of 2008 as well in summer of 2011 when the Swiss Franc strengthened substantially. Industry risk premia also fluctuate with changes in the economic conditions. The results further show that analysts' forecasts of earnings of Swiss firms are biased and inaccurate in volatile times, and I discuss conditions under which this may result in biased estimates of the cost of capital.

\section{Einleitung}

Investoren verlangen für die Bereitstellung von Kapital zur Durchführung von Investitionen eine risikoadjustierte Verzinsung. Da diese Eigenkapitalkosten nicht direkt beobachtbar sind, müssen sie mit Hilfe geeigneter Verfahren empirisch ermittelt werden. Zur Bestimmung der Kapitalkosten können sogenannte implizite Kapitalkostenmodelle verwendet werden, die gerade auch vor dem Hintergrund volatiler Märkte verstärkt Anwendung finden (Bassemir et al. 2012). Dazu wird häufig auf Ergebnisschätzungen von Analysten zurückgegriffen, welche grundsätzlich als verzerrt und ungenau gelten (z. B. Easterwood/

\footnotetext{
* Ich danke zwei anonymen Gutachtern für wertvolle Hinweise.
} 
Nutt 1999; Wallmeier 2005; Bessler/Stanzel 2007) und so auch zu Verzerrungen in den impliziten Kapitalkosten führen können (Easton/Sommers 2007). In diesem Beitrag werden die Eigenkapitalkosten für Schweizer Unternehmen auf monatlicher Basis für den Zeitraum von Januar 2006 bis Juni 2014 geschätzt. Die Ergebnisse zeigen, dass implizite Kapitalkosten konjunkturelle Schwankungen in der Schweiz zeitgerecht abbilden können. Weiterhin werden Genauigkeit und Verzerrung der Analystenschätzungen über ebendiesen Zeitraum ermittelt und gezeigt, dass diese Prognosen auch in der Schweiz im Durchschnitt verzerrt und ungenau sind.

Eigenkapitalkosten sind in vielen finanzwirtschaftlichen Fragestellungen wie der Investitions- und der Bewertungstheorie, aber auch in der Rechnungslegung und der Unternehmenssteuerung von zentraler Bedeutung, um den Wert einer Investition bestimmen zu können. In der Finanzwirtschaft werden Eigenkapitalkosten vorrangig zur Bewertung von ganzen Unternehmen oder einzelnen Investitionsprojekten benötigt. Mit Hilfe der Eigenkapitalkosten werden die zukünftig erwarteten finanziellen Überschüsse eines Unternehmens risikoadäquat diskontiert (z. B. Volkart 2010). In ähnlicher Weise finden die Eigenkapitalkosten auch in der Rechnungslegung Verwendung. So ist die regelmässige Überprüfung der Werthaltigkeit eines derivativ erworbenen Geschäfts- oder Firmenwertes (Goodwill) in der internationalen (IAS 36) und der nationalen (SWISS GAAP FER 20) Rechnungslegung Pflicht, wobei die Entscheidung über die Werthaltigkeit des Goodwill auf einer Unternehmensbewertung mit Hilfe gängiger Bewertungsmethoden basiert. Weiterhin werden Kapitalkosten im Rahmen der wertorientierten Steuerung verwendet. Dabei hat ein Unternehmen in der Periode nur dann einen Wertzuwachs für seine Anteilseigner generiert, wenn mindestens deren geforderte Eigenkapitalrendite - in Form der Verzinsung des Buchwertes des Eigenkapitals mit den Eigenkapitalkosten - erzielt werden konnte (z. B. Stewart 1991). Sowohl bei der Bewertung als auch bei der Steuerung von Unternehmen oder Projekten wird eine zukunftsorientierte Sichtweise eingenommen, um die Wirkung von Entscheidungen abschätzen zu können. Analog zu den finanziellen Überschüssen aus der Planungsrechnung sollten dann auch für die Eigenkapitalkosten die aktuellen Erwartungen der Eigenkapitalgeber in der Entscheidung berücksichtigt werden (Gebhardt/Mansch 2005; Daske/Gebhardt 2006; Bassemir et al. 2012).

Solche zukunftsorientierten Eigenkapitalkosten lassen sich mit Hilfe impliziter Verfahren ermitteln. Unter Berücksichtigung des aktuellen Aktienkurses und der verfügbaren Markterwartungen über die finanziellen Überschüsse eines Unternehmens wird ein Bewertungsmodell invertiert, um die darin implizierten Kapitalkosten zu ermitteln (z. B. Easton 2009). Bisherige Methoden zur Bestimmung der Eigenkapitalkosten welche auf historische Renditen abstellten, wurden als „unavoidably imprecise“ (Fama/French 1997, 153) beschrieben. Vor dem Hintergrund der jüngsten Finanzmarktkrisen zeigte sich ausserdem, dass implizite Kapitalkosten besser in der Lage sind, gestiegene Unsicherheiten unter den Marktteilnehmern auch in gestiegenen Risikoprämien widerzuspiegeln (Bassemir et al. 2012), was mit realisierten Renditen nicht gelingt (vgl. für die Schweiz Pictet \& Cie SA 2015). Trotz dieser Vorteile bestehen gerade bei der Operationalisierung von impliziten Kapitalkosten erhebliche Probleme. So sind die meist als Proxy für die Ergebniserwartungen aller Marktteilnehmer verwendeten Analystenschätzungen vielfach ungenau und verzerrt, was ebenfalls zu verzerrten Kapitalkostenwerten führen dürfte (z. B. Easton 2009 für einen Überblick). 
In diesem Beitrag werden die Eigenkapitalkosten für Schweizer Unternehmen auf der Basis von Analystenprognosen errechnet sowie eine Analyse der Verzerrung und Genauigkeit der Analystenschätzungen vorgenommen. Zur Berechnung der Eigenkapitalkosten wird eine Modifikation des Modells von Gebhardt et al. (2001) (GLS) verwendet, welches eine tagesaktuelle Schätzung der Eigenkapitalkosten erlaubt (Daske et al. 2006). Die Ergebnisse zeigen, dass die Schweizer Marktrisikoprämie im Zuge der Finanzmarktkrise in 2008 und 2009 einen deutlichen Anstieg verzeichnete. Ein erneuter Anstieg ist ab Mitte 2011 mit der zunehmenden Stärke des Schweizer Frankens zu beobachten, welcher die exportorientierte Schweizer Wirtschaft vor neue Herausforderungen stellte. Die Analyse einzelner Branchen zeigt ausserdem, dass sich unterschiedliche Sensitivitäten gegenüber konjunkturellen Faktoren auch in den impliziten Branchenrisikoprämien niederschlagen. So ist der Anstieg der Risikoprämie der Finanzbranche in den Finanzkrisenjahren deutlich stärker als im Zusammenhang mit der zunehmenden Stärke des Frankens. Umgekehrt verhält es sich bei der stark exportorientierten Pharmabranche.

Eine genauere Betrachtung der Analystenprognosen als wichtiger Einflussfaktor auf die Kapitalkosten zeigt jedoch, dass diese gerade in den Zeiten volatiler Aktienmärkte sehr ungenau sind und somit ebenfalls zu verzerrten Eigenkapitalkosten führen können. In den analysierten Jahren sind die Analystenprognosen meist über alle Prognosehorizonte optimistisch verzerrt, für kurzfristige Prognosehorizonte ist allerdings in den Krisenjahren eine pessimistische Verzerrung der Analystenschätzungen zu beobachten. Weiterhin sind Analystenschätzungen gerade für kurze Prognosehorizonte deutlich weniger verzerrt und genauer als Prognosen basierend auf einfachen Fortschreibungen des letzten Ergebnisses. Vor diesem Hintergrund wird diskutiert, ob die bestehenden Alternativen zu den Analystenschätzungen in Zeiten volatiler Märkte zu besseren Ergebnisschätzungen führen können. Wie stark sich die Verzerrung der Analystenschätzungen auf die Eigenkapitalkosten von Schweizer Unternehmen auswirkt, liegt jedoch ausserhalb des Fokus dieses Beitrages.

Der vorliegende Artikel trägt zu einem besseren Verständnis des Verhaltens von impliziten Kapitalkostenmodellen in volatilen Aktienmärkten in der Schweiz bei. Die Vorteilhaftigkeit von impliziten Kapitalkostenmodellen gegenüber Kapitalkosten auf der Basis von realisierten Renditen wird gezeigt und diskutiert. Gleichzeitig weist der Artikel auch auf eine Schwäche der impliziten Kapitalkosten hin, die in der Ungenauigkeit der Analystenschätzungen liegt und quantifiziert den Fehler in den Analystenschätzungen für die Schweiz. Ansätze zur Reduktion des Schätzfehlers in den Analystenprognosen werden diskutiert und so Verbesserungspotenziale für die impliziten Kapitalkosten aufgezeigt.

In Kapitel 2 wird die Methodik der impliziten Kapitalkosten beschrieben und verschiedene Ansätze vorgestellt. In Kapitel 3 wird das Modell von Daske et al. (2006) auf den Schweizer Aktienmarkt angewendet. Anschliessend wird in Kapitel 4 die Verzerrung und die Ungenauigkeit der Analystenschätzungen quantifiziert. Der Beitrag schliesst mit einer Zusammenfassung in Kapitel 5.

\section{Methoden zur Ermittlung von Kapitalkosten}

\subsection{Grundlagen}

Um die Eigenkapitalkosten von Unternehmen zukunftsorientiert zu ermitteln, können verschiedene Verfahren angewendet werden. Alle diese Verfahren basieren auf der Invertierung eines Bewertungsmodells, wobei mit Hilfe von Schätzungen über künftige Unterneh- 
mensüberschüsse und aktuellen Aktienkursen ein diesen Werten implizierter Kapitalkostensatz ermittelt wird (z. B. Easton 2009). Durch Aggregation der so ermittelten Kapitalkosten für einen gesamten Markt oder eine Branche kann daraus die Marktrisikoprämie für den Markt oder die Risikoprämie für eine Branche ermittelt werden. ${ }^{1}$ Als Bewertungsverfahren ist grundsätzlich jedes Modell geeignet, das den Wert des Unternehmens durch die Diskontierung zukünftiger finanzieller Überschüsse ermittelt. Meist wird in der Literatur auf Residualgewinnmodelle zurückgegriffen, da Prognosen für Unternehmensergebnisse von Analysten regelmässig erstellt und im Institutional Brokers' Estimate System (IBES) zur Verfügung gestellt werden (Claus/Thomas 2001; Gebhardt et al. 2001; Easton et al. 2002; Daske et al. 2006). Diese Prognosen von Analysten werden dann als Schätzer für die aktuellen Markterwartungen in das Bewertungsmodell eingesetzt. Alternativ besteht eine grosse Anzahl an Literaturbeiträgen, die eine empirische Prognose von Unternehmensergebnissen erlauben (Feltham/Oblson 1996; Fama/French 2000; Fama/French 2006; Hou/ Robinson 2006; Li 2011; Hou et al. 2012; Houlvan Dijk 2014; Li/Mohanram 2014).

Neben den Prognosen für Unternehmensergebnisse werden zur Berechnung des Residualgewinnes zukünftige Buchwerte des Eigenkapitals benötigt. Basierend auf der Grundannahme des Residualgewinnmodells wird der Buchwert des Eigenkapitals über die Clean Surplus Relation fortgeschrieben (Feltham/Oblson 1995; Oblson 1995). Die Clean Surplus Relation ist erfüllt, sofern alle Veränderungen des Eigenkapitals, ausschliesslich der Ein- und Auszahlungen an die Anteilseigner (sofern sie als Anteilseigner agieren), im Unternehmensergebnis erfasst werden (Preinreich 1937; Lücke 1955). Somit ergibt sich das Eigenkapital in Periode $\mathrm{t}$ als die Summe aus dem Eigenkapital in Periode $\mathrm{t}-1$ und dem Unternehmensergebnis in Periode $\mathrm{t}$ abzüglich der in Periode t gezahlten Dividende gemäss Formel (2).

$$
E K_{t}=E K_{t-1}+G_{t}-D i v_{t}
$$

mit:

$\mathrm{EK}_{\mathrm{t}} \quad=$ Buchwert des Eigenkapitals zum Ende der Periode $\mathrm{t}$

$\mathrm{EK}_{\mathrm{t}-1}=$ Buchwert des Eigenkapitals zum Ende der Periode $\mathrm{t}-1$

$\mathrm{G}_{\mathrm{t}} \quad=$ Gewinn in Periode $\mathrm{t}$

Div $_{t} \quad=$ Gezahlte Dividende in Periode $\mathrm{t}$

Die zur Verfügung stehenden Residualgewinnmodelle, welche die Kapitalkosten auf Unternehmensebene ${ }^{2}$ schätzen, unterscheiden sich vorrangig in der Länge des Detailplanungszeitraumes und der verwendeten Wachstumsrate in der ewigen Rente ${ }^{3}$. Im Modell von Claus/Thomas (2001) wird nach einer fünfjährigen Detailplanungsphase in der ewigen

1 Der Tatsache, dass implizite Kapitalkosten auf Unternehmensebene unterstellen, dass der realisierte Marktpreis den Unternehmenswert darstellt und die Bewertung somit obsolet würde (Tautologie), kann durch die Bildung von Markt- und Branchenrisikoprämien entgegnet werden. Vgl. z. B. Gebhardt et al. (2001); Ballwieser (2005).

2 Es bestehen alternative Verfahren, die die Kapitalkosten auf Portfolioebene schätzen und so eine gleichzeitige Schätzung von Kapitalkosten und Wachstumsrate erlauben, vgl. Easton et al. (2002). In diesem Beitrag liegt der Fokus jedoch auf Modellen, welche die Schätzung firmenspezifischer Kapitalkosten erlauben.

3 Es sei hier angemerkt, dass eine der Schwierigkeiten bei der Anwendung des Residualgewinnmodells für die Kapitalkostenermittlung im Fortführungswert liegt. Eine Fehlspezifikation des Modells kann leicht zu verzerrten Kapitalkostenwerten führen. Ebenso haben unterschiedliche Ausprägungen des bedingten 
Rente eine Wachstumsrate in Höhe der Inflationsrate (in ihrem Fall 3\%) berücksichtigt. Hier wird jedoch auf das GLS-Modell zurückgegriffen, bei welchem im Anschluss an die Detailplanungsphase zunächst sichergestellt wird, dass kein Unternehmen langfristig (im „steady state“) eine Überrendite gegenüber vergleichbaren Unternehmen erzielen kann, bevor dann die ewige Rentenphase beginnt. In der Detailplanungsperiode werden explizite Ergebnisschätzungen für die nächsten fünf Jahre verwendet. In der anschliessenden Übergangsperiode entwickelt sich die Rendite des Unternehmens linear hin zur Median-Brancheneigenkapitalrendite. Die letzte Planungsperiode beinhaltet den Wert des ewigen Rentenstroms, welcher bis in die Ewigkeit eine Rendite in Höhe des Medians der Brancheneigenkapitalrendite generiert (Gebhardt et al. 2001).

\subsection{Modifiziertes GLS Modell nach Daske/Gebhardt/Klein (2006)}

Um die impliziten Kapitalkosten basierend auf den zu diesem Zeitpunkt verfügbaren Informationen zu ermitteln, muss sichergestellt werden, dass nur solche Informationen aus der Bilanz berücksichtigt werden, die zu dem Zeitpunkt öffentlich verfügbar waren. Da Jahresabschlussinformationen meist erst einige Monate nach dem Bilanzstichtag zur Verfügung stehen, beziehen sich die Gewinnschätzungen zu Beginn eines Geschäftsjahres häufig auf den Bilanzstichtag des vergangenen Geschäftsjahres. Für ein Unternehmen mit Bilanzstichtag zum 31.12.x1, das seinen Jahresabschluss am 30.03.x2 veröffentlicht, werden zum 31.01.x2 Gewinnschätzungen für das Geschäftsjahr mit Ende zum 31.12.x1 sowie Prognosen für das aktuelle Geschäftsjahr mit Ende zum 31.12.x2 vorliegen. Gleichzeitig liegt der Buchwert des Eigenkapitals zum 01.01.x2 noch nicht vor, sodass eine Eigenkapitalkostenschätzung zum 31.01.x2 nur auf Basis von Prognosen erstellt werden kann und keine tatsächlich realisierten Werte verwendet werden können. Die verzögerte Berichterstattung stellt somit eine Herausforderung für die unterjährige Ermittlung von erwarteten Eigenkapitalkosten dar (Daske et al. 2006). Ein weiteres Problem liegt in der fehlenden unterjährigen Verfügbarkeit von aktuellen Buchwerten für das laufende Geschäftsjahr (Daske et al. 2006). In den meisten Beiträgen werden die Kapitalkosten aus diesem Grund nach Ablauf von ca. vier bis sechs Monaten seit Geschäftsjahresende ermittelt, um sicherzugehen, dass alle notwendigen Informationen den Marktteilnehmern zur Verfügung stehen und verarbeitet wurden (Gebhardt et al. 2001; Easton et al. 2002).

Für viele Anwendungsbereiche von impliziten Kapitalkosten ist es jedoch von Interesse, die Eigenkapitalkosten oder die Marktrisikoprämie auch unterjährig ermitteln zu können, z.B. um den Wert einer Investition zum Stichtag abschätzen zu können. Das Modell von Daske et al. (2006) modifiziert die Bewertungsformel von GLS, um eine tagesaktuelle Schätzung der Kapitalkosten zu erlauben. Dies erfordert eine Anpassung der Bewertungsformel im ersten Jahr der Detailplanungsperiode unter der Berücksichtigung der zu diesem Zeitpunkt verfügbaren Daten aus der Bilanz und der Erfolgsrechnung. Hierzu wird der Buchwert des Eigenkapitals im ersten Jahr - sofern dieser nicht vorliegt, wird er mit Hilfe der Clean Surplus Relation und den vorliegenden Ergebnisprognosen errechnet - unter der Fiktion der linearen Erwirtschaftung des vorhergesagten Unternehmensergebnisses fortgeschrieben. Für jeden Tag im neuen Geschäftsjahr wird der Buchwert des Eigenkapitals demnach um 1/365 des Unternehmensergebnisses erhöht. Das prognostizierte Unternehmensergebnis für das ge-

Vorsichtsprinzips einen starken Einfluss auf den in der Fortführungsphase realisierten Return on Equity. Vgl. hierzu ausführlich Wallmeier (2007) und Wallmeier (2008). 
samte erste Jahr wird anschliessend um den Teil reduziert, der bereits im Eigenkapital berücksichtigt wurde und wird so zu dem verbleibenden Unternehmensergebnis für das restliche Geschäftsjahr (Daske et al. 2006). Somit kann zu jedem Zeitpunkt im Jahr ein Wert für die Kapitalkosten ermittelt werden (siehe Formel (3) nach Daske et al. 2006).

$b v p s_{t}^{\prime}=b v p s_{0}+\left(1+F R O E_{t, 1}\right)^{\frac{\text { days(t, fiscal year end } 1)}{365}}$

$\mathrm{feps}_{\mathrm{t}}^{\prime}=\mathrm{feps}_{1}-\left(\mathrm{bvps}_{\mathrm{t}}^{\prime}-\right.$ bvps $\left._{0}\right)$

mit:

bvps $_{\mathrm{t}}^{\prime}=$ Angepasster Buchwert des Eigenkapitals pro Aktie zum unterjährigen Zeitpunkt t

bvps $_{0} \quad=$ Buchwert des Eigenkapitals pro Aktie zum Zeitpunkt 0

feps $_{\mathrm{t}}^{\prime} \quad=$ Angepasste Ergebnisprognose der Analysten pro Aktie zum unterjährigen Zeitpunkt $\mathrm{t}$

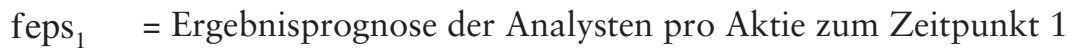

$\mathrm{FROE}_{\mathrm{t}, 1}=$ Prognostizierte Eigenkapitalrendite zum Zeitpunkt $\mathrm{t}$ für das nächste volle Geschäftsjahr nach dem Zeitpunkt t

Die Kapitalkosten aus dem Residualgewinnmodell nach GLS in der Modifikation von Daske et al. (2006) resultieren nun aus der Invertierung der Gleichung in Formel (4) (Formel aus Daske et al. 2006).

$\mathrm{p}_{\mathrm{t}}=\operatorname{bvps}_{\mathrm{t}}^{\prime}+\frac{\text { feps }_{\mathrm{t}}^{\prime}-\left(\left(1+\mathrm{r}_{\mathrm{e}}\right)^{\frac{\text { days(t, fiscal year end } 1)}{365}}-1\right) \cdot \text { bvps }_{\mathrm{t}}^{\prime}}{\left(1+\mathrm{r}_{\mathrm{e}}\right)^{\frac{\text { days(t, fiscal year end } 1)}{365}}}$

$+\sum_{\mathrm{n}=2}^{5} \frac{\text { feps }_{\mathrm{t}, \mathrm{n}}-\mathrm{r}_{\mathrm{e}} \cdot \text { bvps }_{\mathrm{t}, \mathrm{n}-1}}{\left(1+\mathrm{r}_{\mathrm{e}}\right)^{\frac{\text { days(t, fiscal year end } \mathrm{n})}{365}}}$

Detailplanungsperiode

$+\sum_{n=6}^{11} \frac{\left(\mathrm{FROE}_{\mathrm{t}, \mathrm{n}}-\mathrm{r}_{\mathrm{e}}\right) \cdot \mathrm{bvps}_{\mathrm{t}, \mathrm{n}-1}}{\left(1+\mathrm{r}_{\mathrm{e}}\right)^{\frac{\text { days(t, fiscal year end } \mathrm{n})}{365}}}$

Übergangsperiode

$+\frac{\left(\text { FROE }_{t, 12}-r_{\mathrm{e}}\right) \cdot \text { bvps }_{\mathrm{t}, 11}}{\mathrm{r}_{\mathrm{e}} \bullet\left(1+\mathrm{r}_{\mathrm{e}}\right)^{\frac{\text { days }(\mathrm{t}, \text { fiscal year end 11) }}{365}}}$

Endwert

mit:

$\mathrm{p}_{\mathrm{t}} \quad=$ Aktienkurs zum Zeitpunkt $\mathrm{t}$

bvps $_{t}^{\prime}=$ Angepasster Buchwert des Eigenkapitals pro Aktie zum unterjährigen Schätzzeitpunkt $\mathrm{t}$

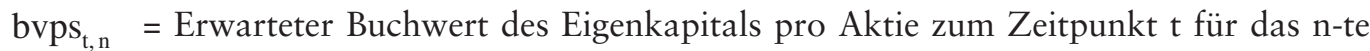
Geschäftsjahr nach dem Schätzzeitpunkt t 
feps $_{\mathrm{t}}^{\prime}=$ Angepasste Ergebnisprognose der Analysten pro Aktie zum unterjährigen Schätzzeitpunkt $\mathrm{t}$

$\mathrm{feps}_{\mathrm{t}, \mathrm{n}}=$ Ergebnisprognose der Analysten pro Aktie zum Zeitpunkt $\mathrm{t}$ für das n-te Geschäftsjahr nach dem Schätzzeitpunkt $t$

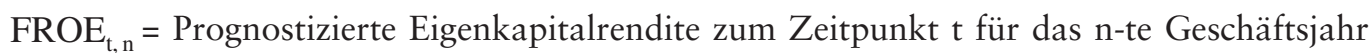
nach dem Schätzzeitpunkt t

$\mathrm{r}_{\mathrm{e}} \quad=$ Impliziter Eigenkapitalkostensatz des Unternehmens

Nach der Schätzung der monatlichen Kapitalkosten pro Unternehmen mit Hilfe des modifizierten GLS-Modells werden die Eigenkapitalkosten aller Schweizer Firmen zu einem Mittelwert aggregiert, um so die implizite Rendite des Marktportfolios zu bestimmen. Von dieser wird anschliessend der risikofreie Zinssatz subtrahiert, um zur Marktrisikoprämie zu gelangen. Unter Berücksichtigung der Unternehmen einer bestimmten Branche werden die Branchenrisikoprämien analog zur Marktrisikoprämie bestimmt (Gebhardt et al. 2001; Daske et al. 2006). Die Risikoprämie einzelner Unternehmen wird in diesem Beitrag nicht bestimmt, kann aber ebenfalls durch Subtraktion des risikofreien Zinssatzes abgeleitet werden.

\section{Kapitalkostenermittlung}

\subsection{Daten und Stichprobe}

Die Stichprobe basiert auf im August 2014 an der SIX Swiss Exchange kotierten Schweizer Firmen. Analystenschätzungen für zukünftige Gewinne werden der IBES-Datenbank ${ }^{4}$ entnommen. Die Daten aus der Rechnungslegung, wie Buchwerte des Eigenkapitals oder gezahlte Dividenden wurden aus Worldscope entnommen. Aktienkurse und Wechselkurse stammen aus Datastream. Die Effektivverzinsung einer 10-jährigen Schweizer Bundesanleihe als Proxy für die risikolose Verzinsung (Loderer et al. 2010; Volkart et al. 2013; Berndt et al. 2014) ist ebenfalls aus Datastream.

\section{Stichprobenauswahl (in Firmenmonaten)}

Ausgangsbasis aller kotierten Schweizer Unternehmen 17.568 mit Analystenschätzungen in IBES

Fehlende Rechnungslegungsdaten (Worldscope), negativer Buchwert des Eigenkapitals oder negatives Ergebnis

Fehlende Analystenschätzungen in IBES oder zu wenige Analysten, die Schätzungen abgeben („Coverage“)

Fehlende Aktienkurse in Datastream

\begin{tabular}{rr}
\hline-2.597 & 14.971 \\
\hline-4.384 & 10.587 \\
\hline-374 & 10.213 \\
\hline
\end{tabular}

Gesamtstichprobe 10.213

Tabelle 1: Stichprobenauswahl

4 Die IBES-Daten wurden über Wharton Research Data Services (WRDS) bezogen und wurden vom Research Priority Program Finance und Financial Markets, Institute for Banking and Finance, Universität Zürich, gesponsert. 
Die Ausgangsbasis der Stichprobe bilden an der SIX kotierte Schweizer Unternehmen, für die Ergebnisschätzungen von Analysten in der IBES-Datenbank vorliegen. Im Zeitraum von Januar 2006 bis Juni 2014 resultiert dies in 17.568 Firmenmonaten. Fehlende Daten aus Worldscope reduzieren den Datensatz um 2.597 Beobachtungen. Zur Schätzung der Kapitalkosten wird weiterhin verlangt, dass mindestens drei Analysten Schätzungen im Firmenmonat abgeben und mindestens Gewinnschätzungen für zwei künftige Perioden in IBES vorliegen. Mit Hilfe der langfristigen Wachstumsrate aus IBES kann dann die Schätzung für die verbleibenden fünf Perioden ermittelt werden. Alternativ besteht die Möglichkeit, die Wachstumsrate aus den bestehenden Prognosen abzuleiten und mit dieser entsprechende Schätzungen selbst zu generieren (Claus/Thomas 2001; Daske et al. 2006). Diese Vorgaben an die IBES Datenbank führen zu einem weiteren Verlust von 4.384 Beobachtungen, sodass die Datenanforderungen für 10.213 Firmenmonate von 155 Unternehmen erfüllt sind.

Bei der Ermittlung der Eigenkapitalkosten gehen noch einmal 101 Beobachtungen verloren, für die keine Kapitalkostenwerte kleiner als 50\% im Rahmen der Invertierung des Bewertungsmodells ermittelt werden können, und so liegen Kapitalkosten basierend auf 10.112 Beobachtungen von 155 Firmen vor.

\subsection{Marktrisikoprämie in der Schweiz}

Abbildung 1 zeigt die monatliche Entwicklung der durchschnittlich erwarteten Rendite am Schweizer Aktienmarkt, der Marktrisikoprämie und des risikolosen Zinssatzes.

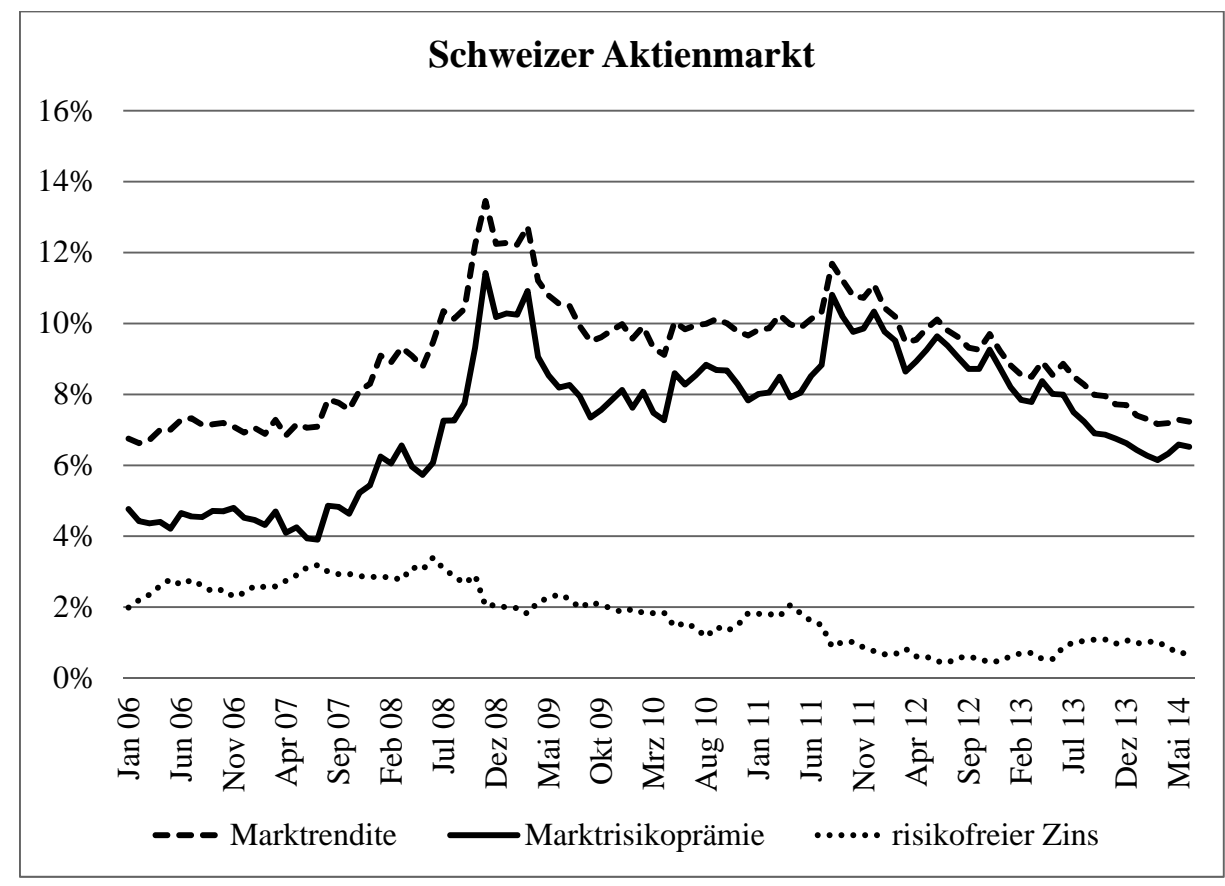

Abbildung 1: Marktrisikoprämie für den gesamten Schweizer Aktienmarkt

Die Abbildung zeigt den Durchschnitt der Eigenkapitalkosten aller Schweizer Aktien pro Monat. Die Risikoprämien werden als Differenz aus den Eigenkapitalkosten und der Effektivverzinsung von Schweizer Bundesobligationen bestimmt. Die Aggregation der unternehmensspezifischen Risikoprämien zu einem monatlichen Durchschnitt aller Schweizer Aktien resultiert in der Marktrisikoprämie. 
Während die historische Marktrisikoprämie in Krisenzeiten aufgrund der damit verbundenen negativen Aktienrenditen eher absinkt (Pictet \& Cie SA 2015), ist die implizite Marktrisikoprämie in der Lage, gestiegene Unsicherheiten am Kapitalmarkt auch in eine höhere Risikoprämie umzuwandeln (Daske et al. 2006; Schneller et al. 2010; Bassemir et al. 2012). Der höchste Wert der Marktrendite und -risikoprämie im gesamten Beobachtungszeitraum kann Ende des Jahres 2008 verzeichnet werden. Dieser Zeitpunkt kennzeichnet mit dem Zusammenbruch der US-amerikanischen Investmentbank Lehman Brothers Inc. den Höhepunkt der Finanzmarktkrise und den Zeitpunkt mit der höchsten Unsicherheit an den internationalen Kapitalmärkten seit 2006 (Schneller et al. 2010). Im weiteren Verlauf ist zunächst die konjunkturelle Erholung seit Mitte 2009 zu beobachten. Die zunehmende Stärke des Schweizer Frankens relativ zu anderen internationalen Währungen wie dem US-Dollar oder dem Euro mit ihrem Höhepunkt im Sommer 2011 ist mit einem erneuten Anstieg der Marktrisikoprämie für den Schweizer Aktienmarkt verbunden. Zusammen mit der europäischen Staatsschuldenkrise stellt die Stärke des Frankens gerade die exportorientierten Unternehmen von Mitte 2011 bis Mitte 2012 vor grosse strukturelle Herausforderungen und führt $\mathrm{zu}$ gestiegenen Unsicherheiten am Schweizer Aktienmarkt (Economiesuisse 2011a; Economiesuisse 2011b). Die konjunkturelle Erholung der Schweizer Wirtschaft seit dem Ende des Jahres 2012 spiegelt sich ebenfalls in einer kontinuierlichen Absenkung der Marktrisikoprämie und der Marktrendite wider. Beide liegen im Juni 2014 wieder in der Nähe des Vorkrisenniveaus zu Beginn des Jahres 2006. Diese Entwicklung zeigt sich auch in den jährlichen Marktrenditen und -risikoprämien in Tabelle 2.

\begin{tabular}{lcccc}
\hline Jahr & Marktrendite & Marktrisikoprämie & risikofreier Zinssatz & N \\
\hline 2006 & $7,02 \%$ & $4,56 \%$ & $2,46 \%$ & 1.189 \\
2007 & $7,44 \%$ & $4,57 \%$ & $2,87 \%$ & 1.223 \\
2008 & $10,31 \%$ & $7,51 \%$ & $2,80 \%$ & 1.350 \\
2009 & $10,80 \%$ & $8,74 \%$ & $2,06 \%$ & 1.201 \\
2010 & $9,77 \%$ & $8,18 \%$ & $1,60 \%$ & 1.110 \\
2011 & $10,48 \%$ & $9,08 \%$ & $1,40 \%$ & 1.256 \\
2012 & $9,72 \%$ & $9,14 \%$ & $0,58 \%$ & 1.165 \\
2013 & $8,36 \%$ & $7,51 \%$ & $0,85 \%$ & 1.079 \\
2014 & $7,26 \%$ & $6,38 \%$ & $0,88 \%$ & 539 \\
\hline Gesamt & $9,16 \%$ & $7,35 \%$ & $1,81 \%$ & 10.112 \\
\hline
\end{tabular}

Tabelle 2: Durchschnittliche jährliche Marktrisikoprämie

\subsection{Branchenrisikoprämien in der Schweiz}

Da für die Bewertung von Unternehmen nicht nur die Höhe der Marktrisikoprämie von Relevanz ist, sondern auch das branchenspezifische Risiko, werden im Folgenden die impliziten Renditen und Risikoprämien einzelner Branchen analysiert. Tabelle 3 zeigt eine jährliche Übersicht der Risikoprämien in verschiedenen Schweizer Branchen. Hohe erwartete Renditen sind in stark konjunkturabhängigen Branchen zu beobachten (Gebhardt et al. 2001; Daske et al. 2006; Bassemir et al. 2012). So weisen der Einzelhandel, die Metall- 
und die Öl- und Gasindustrie, der Tourismus, das Druck und Verlagswesen und die Finanzindustrie über den gesamten Zeitraum durchschnittliche Risikoprämien von über $8 \%$ auf. Im Gegensatz dazu weisen Industrien wie Pharma, Transport, Chemie und Bekleidung / Textilien relativ geringe Risikoprämien auf, welche im Durchschnitt des gesamten Beobachtungszeitraumes unter 6,5\% liegen.

Für alle Branchen ist ein deutlicher Anstieg der Eigenkapitalkosten im Zuge der Finanzmarktkrise im Jahr 2008 und teilweise auch im Jahr 2009 zu beobachten. Im Gegensatz dazu wirkten sich die Stärke des Frankens und die europäische Staatsschuldenkrise, welche die Marktrisikoprämie von Mitte 2011 bis Mitte 2012 deutlich erhöhten, nicht in allen Branchen gleich stark aus. Vor allem exportorientierte Branchen wie Chemie und Pharma oder stark konjunkturabhängige Branchen wie Tourismus, Einzelhandel oder Textilien hatten mit einem erheblichen Anstieg ihrer Eigenkapitalkosten von 2010 auf 2011 zu kämpfen. Seit dem Jahr 2013 sanken die Eigenkapitalkosten jedoch in allen Schweizer Branchen wieder und nähern sich zur Jahresmitte 2014 grösstenteils dem Vorkrisenniveau von 2006 an.

\begin{tabular}{lccccccccccc}
\hline Branche & 2006 & 2007 & 2008 & 2009 & 2010 & 2011 & 2012 & 2013 & 2014 & $\phi$ & $\mathrm{N}$ \\
\hline Bau & $5.7 \%$ & $6,6 \%$ & $6,9 \%$ & $7,5 \%$ & $8,2 \%$ & $9,1 \%$ & $8,2 \%$ & $6,9 \%$ & $6,2 \%$ & $7,4 \%$ & 466 \\
Bekleidung & $2.2 \%$ & $0,9 \%$ & $3,7 \%$ & $8,9 \%$ & $6,2 \%$ & $5,5 \%$ & $7,4 \%$ & $8,8 \%$ & & $4,7 \%$ & 43 \\
Chemie & $4.0 \%$ & $3,7 \%$ & $5,3 \%$ & $4,9 \%$ & $5,4 \%$ & $6,2 \%$ & $6,8 \%$ & $5,7 \%$ & $4,8 \%$ & $5,2 \%$ & 596 \\
Druck \& & & & & & & & & & & & \\
Verlagswesen & $3.8 \%$ & $5,6 \%$ & $9,0 \%$ & $13,1 \%$ & $11,6 \%$ & $11,0 \%$ & $10,4 \%$ & $9,6 \%$ & $8,9 \%$ & $8,4 \%$ & 120 \\
Einzelhandel & $9.7 \%$ & $8,4 \%$ & $8,6 \%$ & $9,2 \%$ & $5,6 \%$ & $6,9 \%$ & $8,3 \%$ & $6,9 \%$ & $5,4 \%$ & $8,0 \%$ & 267 \\
Elektrik & $3.8 \%$ & $3,5 \%$ & $7,5 \%$ & $9,1 \%$ & $8,5 \%$ & $7,6 \%$ & $7,5 \%$ & $5,6 \%$ & $5,1 \%$ & $6,7 \%$ & 286 \\
Elektronik & $2.7 \%$ & $3,2 \%$ & $7,5 \%$ & $8,1 \%$ & $6,7 \%$ & $9,9 \%$ & $11,2 \%$ & $11,9 \%$ & $11,0 \%$ & $7.7 \%$ & 603 \\
Finanzen & $5.6 \%$ & $5,4 \%$ & $9,0 \%$ & $9,7 \%$ & $9,4 \%$ & $9,6 \%$ & $10,0 \%$ & $7,8 \%$ & $7,1 \%$ & $8,3 \%$ & 2.058 \\
Gemischt & $3.5 \%$ & $3,7 \%$ & $8,9 \%$ & $13,0 \%$ & $10,2 \%$ & $11,9 \%$ & $9,8 \%$ & $7,3 \%$ & $6,7 \%$ & $8,5 \%$ & 362 \\
Maschinen \& & $3.8 \%$ & $3,6 \%$ & $6,4 \%$ & $8,4 \%$ & $7,7 \%$ & $9,0 \%$ & $8,8 \%$ & $7,4 \%$ & $5,8 \%$ & $6,6 \%$ & 1.360 \\
Zubehör & & & & & & & & & & & \\
Metallpro- & & & & & & & & & & \\
duktherstel- & $2.2 \%$ & $5,2 \%$ & $8,9 \%$ & $11,1 \%$ & $9,3 \%$ & $9,1 \%$ & $10,1 \%$ & $8,9 \%$ & $7,3 \%$ & $8,4 \%$ & 281 \\
lung & & & & & & & & & & & \\
$\begin{array}{l}\text { Metall- } \\
\text { produktion }\end{array}$ & & $16,8 \%$ & $20,6 \%$ & $22,9 \%$ & $20,9 \%$ & $10,1 \%$ & $11,1 \%$ & $10,3 \%$ & & $16,5 \%$ & 51 \\
$\begin{array}{l}\text { Nahrungs- } \\
\text { mittel }\end{array}$ & $6.6 \%$ & $5,5 \%$ & $6,9 \%$ & $8,6 \%$ & $8,6 \%$ & $8,4 \%$ & $8,4 \%$ & $7,1 \%$ & $5,9 \%$ & $7,4 \%$ & 559 \\
Öl, Gas, Kohle & & $20,9 \%$ & $26,2 \%$ & $15,2 \%$ & & & & & & $23,6 \%$ & 21 \\
Pharma & $3.9 \%$ & $3,7 \%$ & $5,8 \%$ & $7,4 \%$ & $7,5 \%$ & $9,1 \%$ & $8,0 \%$ & $5,8 \%$ & $4,4 \%$ & $6,3 \%$ & 973 \\
Sonstige & $4.4 \%$ & $4,1 \%$ & $7,2 \%$ & $8,8 \%$ & $8,3 \%$ & $8,7 \%$ & $9,2 \%$ & $7,6 \%$ & $6,4 \%$ & $7,3 \%$ & 1.543 \\
Textilien & & $-0,7 \%$ & $1,4 \%$ & $4,3 \%$ & $4,1 \%$ & $8,0 \%$ & $9,5 \%$ & $10,7 \%$ & $10,1 \%$ & $6,0 \%$ & 85 \\
Tourismus & $0.6 \%$ & $0,4 \%$ & $2,5 \%$ & $2,2 \%$ & $8,9 \%$ & $14,0 \%$ & $13,8 \%$ & $12,5 \%$ & $10,4 \%$ & $10,5 \%$ & 131 \\
Transport & $0.0 \%$ & $-0,2 \%$ & $1,3 \%$ & $1,5 \%$ & $1,6 \%$ & $2,2 \%$ & $2,3 \%$ & $1,7 \%$ & $1,6 \%$ & $1,3 \%$ & 102 \\
Versorger & $6.7 \%$ & $6,1 \%$ & $7,7 \%$ & $8,2 \%$ & $8,3 \%$ & $9,2 \%$ & $6,5 \%$ & $6,0 \%$ & $5,9 \%$ & $7,3 \%$ & 205 \\
\hline Gesamt & $4,6 \%$ & $4,6 \%$ & $7,5 \%$ & $8,7 \%$ & $8,2 \%$ & $9,1 \%$ & $9,1 \%$ & $7,5 \%$ & $6,4 \%$ & $7,4 \%$ & 10.112 \\
\hline
\end{tabular}

Tabelle 3: Jährliche durchschnittliche Branchenrisikoprämien 
Abbildung 2 zeigt die monatliche Entwicklung der durchschnittlichen Eigenkapitalkosten und Branchenrisikoprämie der Finanzbranche als einer der wichtigsten Schweizer Industriezweige auf. In der Abbildung wird die Anfälligkeit der Finanzbranche für makroökonomische Entwicklungen deutlich. So verzeichnet die Finanzbranche im Zuge des Zusammenbruchs der Investmentbank Lehman Brothers Inc. Ende 2008 und aufgrund der Auswirkungen der europäischen Staatsschuldenkrise, der Stärke des Franken und der damit verbundenen konjunkturellen Eintrübung in Europa und der Schweiz ab Mitte 2011 bis Mitte 2012 hohe Risikoprämien. Durch die internationalen Verflechtungen im Bankenbereich wirkt sich der Zusammenbruch von Lehman Brothers Inc. deutlich stärker auf die Branchenrisikoprämie aus, als die mit der Stärke des Frankens verbundene konjunkturelle Eintrübung ab Mitte des Jahres 2011, die vorrangig die Realindustrie betraf. Die zunehmende Erholung der Schweizer Volkswirtschaft ab Anfang des Jahres 2013 zeigt sich in gesunkenen durchschnittlichen Eigenkapitalkosten der Finanzbranche, die sich im Juni 2014 wieder auf dem gleichen Niveau wie schon in 2006 bewegen.

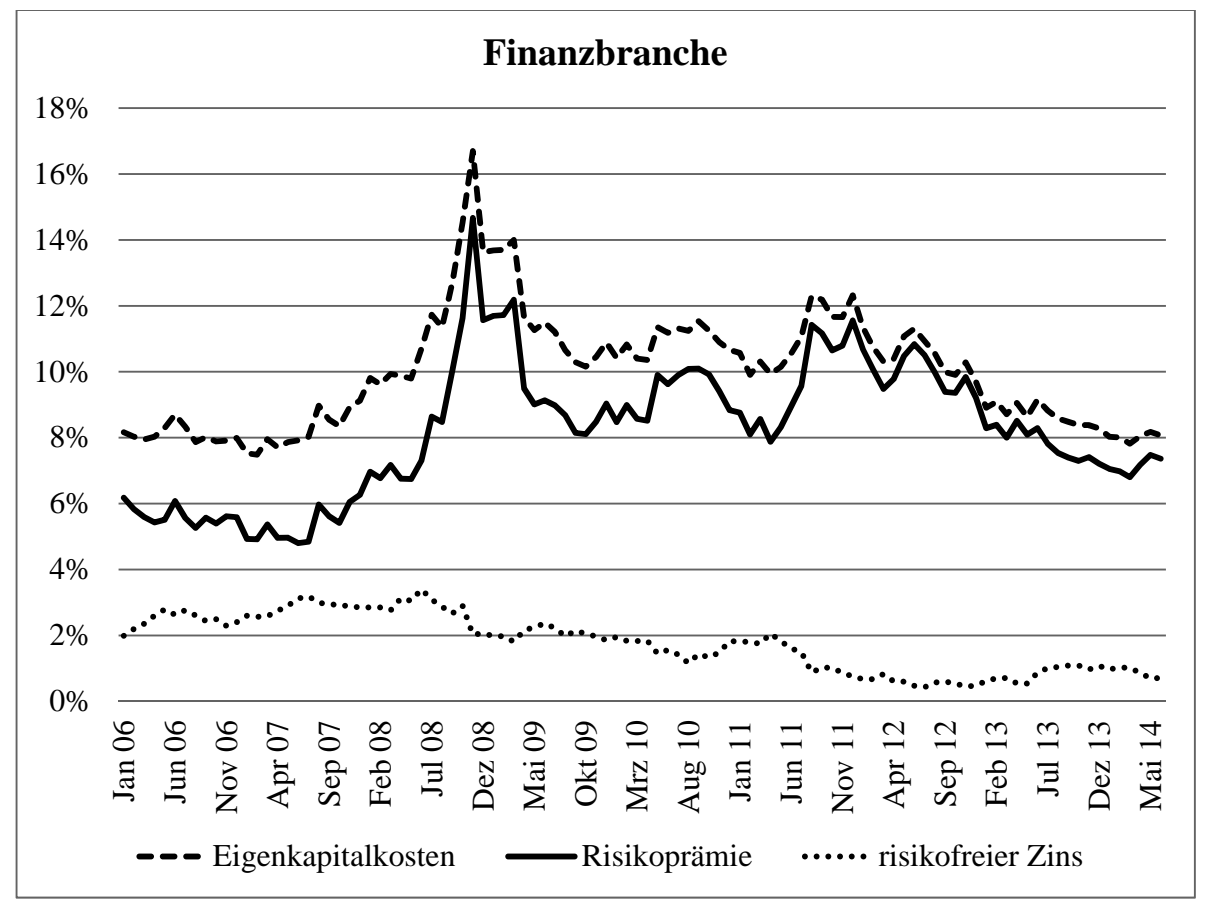

Abbildung 2: Eigenkapitalkosten und Risikoprämie in der Finanzbranche

Die Abbildung zeigt die durchschnittlichen monatlichen Eigenkapitalkosten und die Risikoprämie in der Finanzbranche sowie die monatliche Effektivverzinsung der Schweizer Bundesobligationen als risikofreien Zinssatz.

Als zweiter wichtiger Industriezweig aus der Schweizer Realwirtschaft wird nun die Pharmabranche betrachtet (Abbildung 3). Die geringen Unsicherheiten in der Pharmabranche führen zu einem überaus niedrigen Ausgangsniveau der Eigenkapitalkosten zu Beginn des Jahres 2006. Die verschlechterten Exportaussichten aufgrund der Stärke des Franken ab Mitte 2011 und der damit verbundene Druck auf die Margen führte zu einem extremen Anstieg der durchschnittlichen Eigenkapitalkosten in der Pharmabranche (Economiesuisse 
2011a). Zeitweise lagen diese sogar über 11\%, was gerade vor dem Hintergrund des sehr niedrigen Vorkrisenniveaus von ca. 6\% beachtlich ist. Im Gegensatz dazu wirkte sich die weltweite Finanzkrise um den Zusammenbruch von Lehman Brothers Inc. moderater auf die durchschnittlichen Eigenkapitalkosten der Branche aus. Im Vergleich mit der Finanzbranche wurde die Pharmabranche von der Finanzkrise weniger stark getroffen. Für die Krise in der Eurozone und die damit verbundene Stärke des Frankens gilt jedoch das Gegenteil. Zur Jahresmitte 2014 liegen die durchschnittlichen Eigenkapitalkosten der Branche, aufgrund der wirtschaftlichen Erholung in der Schweiz, mit ca. 5\% wieder auf dem Vorkrisenniveau des Jahres 2006.

Die exemplarische Analyse der Finanz- und der Pharmabranche zeigt, dass die implizite Ermittlung der Kapitalkosten die unterschiedliche Sensitivität der Branchen auf konjunkturelle Einflüsse abzubilden vermag. In Zeiten erhöhter Unsicherheit bzgl. der weiteren wirtschaftlichen Entwicklung einer Branche steigen die durchschnittlichen Eigenkapitalkosten der Unternehmen an. Geht die Unsicherheit zurück, so ist auch ein Absinken der durchschnittlichen Kapitalkosten zu beobachten.

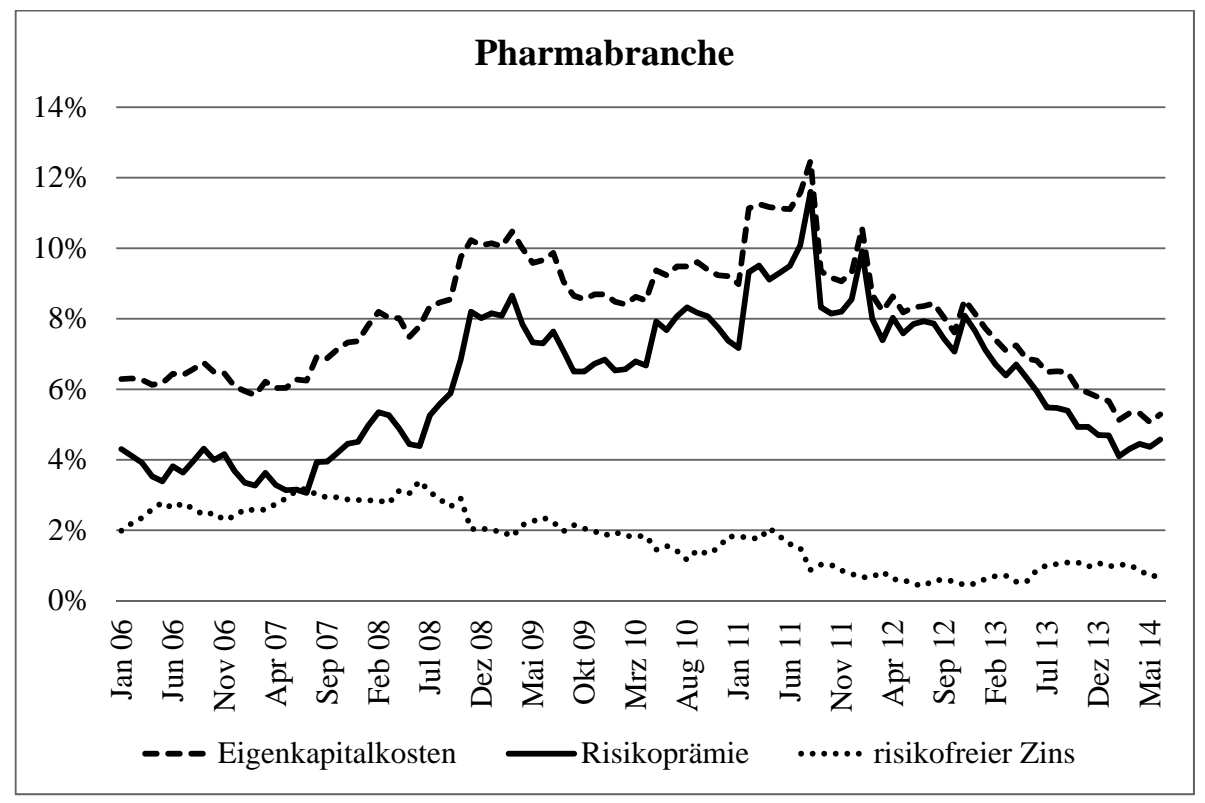

Abbildung 3: Eigenkapitalkosten und Risikoprämie in der Pharmabranche

Die Abbildung zeigt die durchschnittlichen monatlichen Eigenkapitalkosten und Risikoprämie in der Pharmabranche sowie die monatliche Effektivverzinsung der Schweizer Bundesobligationen als risikofreien Zinssatz.

\section{Schätzfehler in den Analystenprognosen}

Trotz der Vorteile der impliziten Kapitalkosten haben diese Methoden auch einige Schwächen. Neben einigen konzeptionellen Schwächen (Gebhardt et al. 2001; Ballwieser 2005; Wallmeier 2007; Wallmeier 2008), liegen diese vorrangig in der empirischen Umsetzung der Modelle (vgl. Easton 2009 für eine Übersicht). So wurde bereits in früheren Beiträgen gezeigt, dass Analysten grundsätzlich zu optimistisch sind und häufig keine Vorhersagen 
treffen, die nahe an den tatsächlich realisierten Gewinnen liegen (Easterwood/Nutt 1999; Bolliger 2004; Wallmeier 2005). Anknüpfend an die Beobachtung, dass Analystenschätzungen optimistisch verzerrt sind, sollten auch die auf Basis dieser Schätzungen ermittelten Werte der impliziten Kapitalkosten verzerrt sein (Claus/Thomas 2001; Easton/Sommers 2007). Diese Beobachtung wird nun für den hier analysierten Zeitraum näher betrachtet. Dabei stellt sich die Frage ob die Analysten auch in der Krise zu optimistisch waren und ob höhere Kapitalkostenwerte möglicherweise aus diesen optimistisch verzerrten Analystenprognosen resultieren könnten. ${ }^{5}$

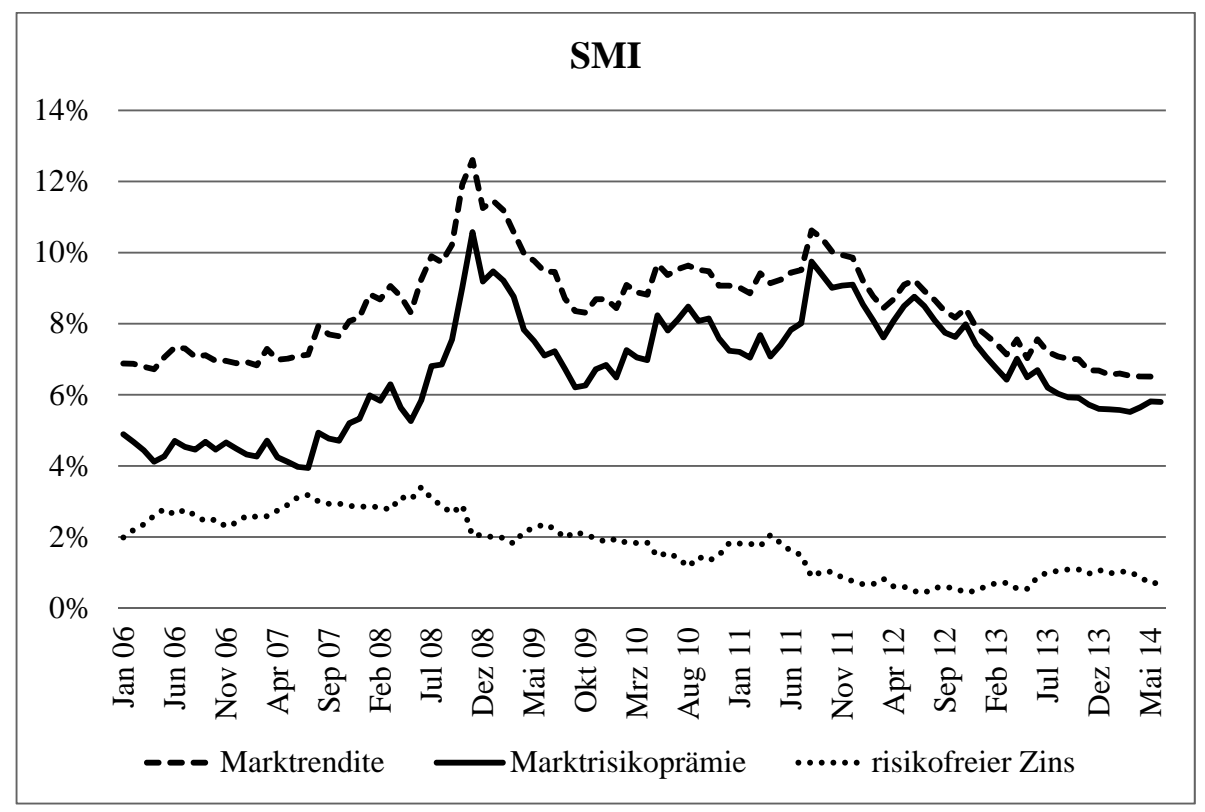

Abbildung 4: Marktrisikoprämie für den SMI

Die Abbildung zeigt die durchschnittlichen monatlichen Eigenkapitalkosten und Risikoprämie der SMI Unternehmen sowie die monatliche Effektivverzinsung der Schweizer Bundesobligationen als risikofreien Zinssatz.

Easton/Sommers (2007) zeigen, dass die impliziten Eigenkapitalkosten für kleinere Firmen meist stärker nach oben verzerrt sind als die Eigenkapitalkosten für grössere Unternehmen. Der Grund dafür liegt in der grösseren optimistischen Verzerrung der Analystenschätzungen für kleinere Unternehmen. Dies führt bei Gleichgewichtung aller Aktien zu einer nach oben verzerrten Marktrisikoprämie. Um das Ausmass dieser Verzerrung in einem ersten Schritt abschätzen zu können, zeigt Abbildung 4 die Marktrisikoprämie als gleichgewichteten Durchschnitt der grössten - im Swiss Market Index (SMI) kotierten Schweizer Firmen. Der Verlauf der Marktrisikoprämie ist analog zur Entwicklung der Marktrisikoprämie für den Schweizer Gesamtmarkt. Die Marktrisikoprämie basierend auf den Eigenkapitalkosten der SMI Firmen liegt lediglich auf einem ca. einen Prozentpunkt niedrigeren Niveau als die Marktrisikoprämie des Gesamtmarktes.

5 Um eine konkrete Aussage über den Schätzfehler in den Kapitalkosten treffen zu können, müssten die impliziten Kapitalkosten evaluiert werden; vgl. hierzu die Verfahren in Botosan/Plumlee (2005); Easton/Monahan (2005); Lee et al. (2014). 
Zur Quantifizierung des Fehlers in den Analystenprognosen werden nun die Verzerrung und die Genauigkeit dieser Schätzungen ermittelt. Die Verzerrung wird dabei als Differenz zwischen den realisierten Gewinnen je Aktie und den Prognosen der Analysten für die Gewinne je Aktie ermittelt (Easterwood/Nutt 1999; Capstaff et al. 2001). Dieser Wert wird anschliessend mit dem Aktienkurs zum Zeitpunkt der Veröffentlichung der Prognose skaliert (Brown 1996; Easterwood/Nutt 1999). ${ }^{6}$ Die monatlich verfügbaren Aktualisierungen der Analystenschätzungen werden dann als Mittelwert pro Vorhersagejahr aggregiert. Zum Vergleich wird ausserdem der Prognosefehler bei Verwendung des aktuellsten Unternehmensergebnisses als Vorhersagewert berechnet (sogenannte Random Walk (RW) Prognose, Bradshaw et al. 2012).

$$
\begin{aligned}
R F_{n, T} & =\frac{e p s_{n, T}-\text { feps }_{n, t, T}}{\text { price }_{n, t}} \\
\mathrm{RAF}_{\mathrm{n}, \mathrm{T}} & =\frac{\mid \operatorname{eps}_{\mathrm{n}, \mathrm{T}}-\text { feps }_{\mathrm{n}, \mathrm{t}, \mathrm{T}} \mid}{\text { price }_{\mathrm{n}, \mathrm{t}}}
\end{aligned}
$$

mit:

$\mathrm{RF}_{\mathrm{n}, \mathrm{T}} \quad=$ Relativer Fehler (Verzerrung) in den Prognosen für Unternehmen $\mathrm{n}$ für das Geschäftsjahr T

$\mathrm{RAF}_{\mathrm{n}, \mathrm{T}}=$ Relativer absoluter Fehler (Genauigkeit) in den Prognosen für Unternehmen $\mathrm{n}$ für das Geschäftsjahr T

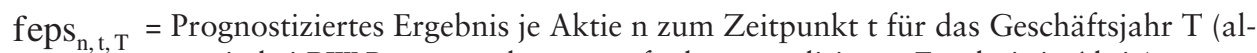
ternativ bei RW-Prognose: letztes verfügbares realisiertes Ergebnis je Aktie)

eps $_{\mathrm{n}, \mathrm{T}} \quad=$ Realisiertes Ergebnis je Aktie $\mathrm{n}$ im Geschäftsjahr T

price $_{n, t}=$ Aktienkurs der Aktie n zum Prognosezeitpunkt $\mathrm{t}$

Tabelle 4 zeigt, dass die Analysten im Mittel über den gesamten Zeitraum zu optimistisch sind und dies unabhängig vom Prognosehorizont. Tendenziell steigt die Stärke der Verzerrung für längere Vorhersagehorizonte an. Interessanterweise sind aber gerade die kurzfristigen Prognosen im Vorkrisenzeitraum 2006 und 2007 sowie im Jahr 2009 pessimistisch verzerrt. Die Erwartungen der Analysten für diese Jahre waren also geringer als sie schlussendlich realisiert wurden. Im Gegensatz dazu sind die kurzfristigen Erwartungen in 2008 sowie ab 2010 wieder optimistisch verzerrt, was im Einklang mit bisherigen Studien ist (Bolliger 2004; Wallmeier 2005). Vergleicht man die Verzerrung der Analystenprognosen mit einer einfachen Fortschreibung des aktuellen Ergebnisses (Tabelle 5), so zeigt sich, dass für einjährige Prognosen Analystenschätzungen in den Krisenjahren sogar weniger stark verzerrt sind als bei einfacher Ergebnisfortschreibung. Für langfristigere Prognosehorizonte kehrt sich dieser Effekt jedoch wieder um (siehe für Ergebnisse mit ähnlicher Tendenz am deutschen Markt, Bessler/Stanzel 2007).

6 In Zeiten volatiler Aktienkurse besteht die Gefahr, dass die relativen Fehler in den Analystenschätzungen stark mit dem Aktienkurs schwanken (Bessler/Stanzel 2007). Alternativ zeigen Tabellen 8 und 9 im Anhang die Ergebnisse für die Verzerrung und Genauigkeit bei einer Skalierung mit dem Absolutwert des realisierten Ergebnisses je Aktie (Capstaff et al. 1998; Wallmeier 2005). Diese Skalierung führt zu sehr ähnlichen Implikationen wie die Skalierung mit dem Aktienkurs, hat jedoch den Nachteil, dass ca. $25 \%$ der Beobachtungen verloren gehen, weil die realisierten Ergebnisse zu klein sind und so zu relativen Fehlern grösser als 1 führen, welche aus der Stichprobe eliminiert werden (Capstaff et al. 1998; Wallmeier 2005). 


\begin{tabular}{|c|c|c|c|c|c|}
\hline Jahr & $\boldsymbol{\phi R F}_{\mathbf{J a h r}+1}$ & $\phi \mathbf{R F}_{\mathbf{J a h r}+2}$ & $\boldsymbol{\phi R F}_{\mathbf{J a h r}+3}$ & $\phi \mathbf{R F}_{\mathbf{J a h r}+4}$ & $\phi \mathbf{R F}_{\mathbf{J a h r}+5}$ \\
\hline 2006 & $0,00381 * *$ & $0,00453 *$ & $-0,02271 * * *$ & $-0,03924 * * *$ & $-0,03574 * * *$ \\
\hline 2007 & $0,00305 * *$ & $-0,02971 * * *$ & $-0,04845 * *$ & $-0,04631 * * *$ & $-0,03799 * *$ \\
\hline 2008 & $-0,03309 * * *$ & $-0,06514 * * *$ & $-0,05892 * * *$ & $-0,05222 * * *$ & $-0,06450 * * *$ \\
\hline 2009 & 0,00191 & $-0,01896 * *$ & $-0,02803 * * *$ & $-0,03703 * * *$ & $-0,04368 * * *$ \\
\hline 2010 & $-0,00423 * *$ & $-0,02193 * * *$ & $-0,03324 * * *$ & $-0,03309 * * *$ & $-0,03853 * * *$ \\
\hline 2011 & $-0,02279 * * *$ & $-0,04101 * * *$ & $-0,04955 * * *$ & $-0,03362 * * *$ & \\
\hline 2012 & $-0,02088 * * *$ & $-0,03178 * * *$ & $-0,04212 * * *$ & & \\
\hline 2013 & $-0,01432 * * *$ & $-0,01881 * * *$ & & & \\
\hline 2014 & $-0,00589 * *$ & & & & \\
\hline Gesamt & $-0,01109 * * *$ & $-0,02970 * * *$ & $-0,04078 * * *$ & $-0,04024 * * *$ & $-0,04650 * * *$ \\
\hline
\end{tabular}

Tabelle 4: Durchschnittliche jährliche Verzerrung der Analystenprognosen

Für jeden Prognosehorizont (in Jahren und nicht in Monaten, hier: Jahr $+n, n=1, \ldots, 5$ ) werden die mittleren relativen Fehler für alle Schweizer Aktien ermittelt und zu einem Durchschnitt pro Jahr aggregiert. Die Zeile „Gesamt“ beinhaltet den Durchschnitt der mittleren relativen Fehler über den Gesamtzeitraum pro Prognosehorizont. Die Sterne zeigen die Signifikanz der einseitigen t-Tests (Mittelwert von null). * $10 \%$, $* * 5 \%, * * 1 \%$.

\begin{tabular}{|c|c|c|c|c|c|}
\hline Jahr & $\phi \mathbf{R F}_{\mathrm{Jahr}+1}$ & $\phi \mathbf{R F}_{\mathrm{Jahr}+2}$ & $\phi \mathbf{R F}_{\mathrm{Jahr}+3}$ & $\phi \mathbf{R F}_{\mathrm{Jahr}+4}$ & $\phi \mathrm{RF}_{\mathrm{Jahr}+5}$ \\
\hline 2006 & $0,01808 * * *$ & $0,02996 * * *$ & 0,01154 “ & $-0,00671^{\prime \alpha}$ & $-0,00049$ “ \\
\hline 2007 & $0,01082 * * *$ & $-0,01106 ، “$ & -0,01899““ & $-0,01532$ “ & $-0,00577$ “ \\
\hline 2008 & $-0,04089 * * *$ & $-0,06100 “$ & $-0,04219$ “ & $-0,03807$ ““ & $-0,04599$ ““ \\
\hline 2009 & $-0,04937 * * *$ & $-0,04844 * * *$ & $-0,03799 * * *$ & $-0,03037^{،}$ & $-0,02636$ “ \\
\hline 2010 & $-0,01730 * * *$ & $-0,02006$ & $-0,01889$ “ & $-0,01318$ “ & $-0,02259^{\prime}$ \\
\hline 2011 & $-0,02056$ & $-0,02360$ “ & $-0,01916$ “ & 0,00046“ & \\
\hline 2012 & $-0,01882$ & $-0,01724$ “ & $-0,02732$ “ & & \\
\hline 2013 & $-0,00789$ “ & $-0,02075$ & & & \\
\hline 2014 & $-0,00086$ “ & & & & \\
\hline Gesamt & $-0,01596 * * *$ & $-0,02250$ “ & $-0,02183^{\prime \omega}$ & $-0,01981$ “ & $-0,02473$ “ \\
\hline
\end{tabular}

\section{Tabelle 5: Durchschnittliche jährliche Verzerrung der RW-Prognose}

Für jeden Prognosehorizont (in Jahren und nicht in Monaten, hier: Jahr $+n, n=1, \ldots, 5$ ) werden die mittleren relativen Fehler für alle Schweizer Aktien ermittelt und zu einem Durchschnitt pro Jahr aggregiert. Die Zeile "Gesamt“ beinhaltet den Durchschnitt der mittleren relativen Fehler über den Gesamtzeitraum pro Prognosehorizont. Die Sterne zeigen die Signifikanz der einseitigen t-Tests (Mittelwert von null) sofern die Werte in Tabelle 5 grösser sind als in Tabelle 4 während die Apostrophe die Signifikanz für Werte in Tabelle 5 die kleiner sind als in Tabelle 4 zeigen. $* / * 10 \%, * * / “ 5 \%, * * * / \cdots 1 \%$.

Die Analyse der Genauigkeit der Analystenschätzungen ermöglicht es ein besseres Gesamtbild über die Höhe des Fehlers in den Schätzungen zu erhalten. Tabelle 6 zeigt, dass der Fehler in den Prognosen der Analysten im Markt in den Krisenjahren 2008 und 2009 für alle Prognosehorizonte deutlich über dem Durchschnitt über alle hier betrachteten Perioden liegt. Fehler in den Analystenprognosen scheinen also gerade in den Zeiträumen der Krise besonders deutlich zu sein, was sich in ungenaueren Kapitalkostenwerten widerspie- 
geln könnte. Nichtsdestotrotz sind die Analystenprognosen in diesen beiden Jahren im Vergleich mit der naiven Extrapolation des letzten Ergebnisses (RW-Prognose) für kurze Zeiträume deutlich genauer. Für längere Prognosehorizonte kehrt sich dieser Effekt in der Tendenz um (siehe für Ergebnisse mit ähnlicher Tendenz am amerikanischen Markt, Bradshaw et al. 2012 und am deutschen Markt, Bessler/Stanzel 2007).

\begin{tabular}{|c|c|c|c|c|c|}
\hline Jahr & $\phi \mathbf{R A F}_{\mathrm{Jahr}+1}$ & $\phi \mathbf{R A F}_{\mathrm{Jahr}+2}$ & $\phi \mathbf{R A F}_{\text {Jahr }+3}$ & $\phi \mathbf{R A F}_{\mathrm{Jahr}+4}$ & $\phi \mathbf{R A F}_{\mathrm{Jahr}+5}$ \\
\hline 2006 & $0,02344 * * *$ & $0,04501^{* * *}$ & $0,06186^{* * *}$ & $0,07041^{* * * *}$ & $0,04543 * * *$ \\
\hline 2007 & $0,02414 * * *$ & $0,05513 * * *$ & $0,06696^{* * *}$ & $0,05148 * * *$ & $0,04133 * * *$ \\
\hline 2008 & $0,05536 * * *$ & $0,08928 * * *$ & $0,06782^{* * *}$ & $0,06832 * *$ & $0,07811 * * *$ \\
\hline 2009 & $0,05006^{* * *}$ & $0,06099 * * *$ & $0,06776^{* * *}$ & $0,07193 * *$ & $0,07222^{* * * *}$ \\
\hline 2010 & $0,02529 * * *$ & $0,04244 * * *$ & $0,04874 * * *$ & $0,05061 * * *$ & $0,05630 * * *$ \\
\hline 2011 & $0,03821 * *$ & $0,05398 * *$ & $0,05820^{* * *}$ & $0,04471^{* * * *}$ & \\
\hline 2012 & $0,03429 * * *$ & $0,04393 * *$ & $0,05447^{* * *}$ & & \\
\hline 2013 & $0,02256 * * *$ & $0,03022^{* * *}$ & & & \\
\hline 2014 & $0,01324 * * *$ & & & & \\
\hline Gesamt & $0,03438 * * *$ & $0,05602 * * *$ & $0,06193 * * *$ & $0,06242 * * *$ & $0,06511^{* * *}$ \\
\hline
\end{tabular}

Tabelle 6: Durchschnittliche jährliche Genauigkeit von Analystenprognosen

Für jeden Prognosehorizont (in Jahren und nicht in Monaten, hier: Jahr $+n, n=1, \ldots, 5$ ) werden die mittleren relativen absoluten Fehler für alle Schweizer Aktien ermittelt und zu einem Durchschnitt pro Jahr aggregiert. Die Zeile „Gesamt“ beinhaltet den Durchschnitt der mittleren relativen absoluten Fehler über den Gesamtzeitraum pro Prognosehorizont. Die Sterne zeigen die Signifikanz der einseitigen t-Tests (Mittelwert von null). $* 10 \%, * * 5 \%, * * 1 \%$.

\begin{tabular}{|c|c|c|c|c|c|}
\hline Jahr & $\boldsymbol{\phi R} \mathbf{A} \mathbf{F}_{\mathbf{J a h r}}+1$ & $\phi \mathbf{R A F}_{\mathbf{J a h r}}+2$ & $\phi \mathbf{R A F}_{\text {Jahr }+3}$ & $\phi \mathbf{R A F}_{\text {Jahr }+4}$ & $\phi \mathbf{R A F}_{\mathrm{Jahr}+5}$ \\
\hline 2006 & $0,02981 * * *$ & $0,05384 * * *$ & $0,05891^{\prime}$ & 0,06359““ & 0,03355 “ \\
\hline 2007 & 0,02399 & $0,05261^{`}$ & 0,05195 “ & 0,03538“ & 0,02606 “ \\
\hline 2008 & $0,06655 * *$ & $0,09395 * *$ & $0,06495^{\circ}$ & 0,05642، & 0,06768 “ \\
\hline 2009 & $0,09372 * * *$ & $0,08061 * * *$ & $0,07907 * \ldots$ & 0,07238 & 0,06929 \\
\hline 2010 & $0,04960 * * *$ & $0,05309 * * *$ & 0,05222 & 0,05472 & 0,05767 \\
\hline 2011 & $0,04380 * * *$ & 0,04953““ & 0,04609 “ & 0,03460“ & \\
\hline 2012 & $0,04276 * * *$ & 0,04379 & 0,05506 & & \\
\hline 2013 & $0,02623 * *$ & $0,04533 * *$ & & & \\
\hline 2014 & $0,02010 * * *$ & & & & \\
\hline Gesamt & $0,04712 * * *$ & $0,06139 * * *$ & 0,05895 “ & 0,05750 “ & 0,05804 “ \\
\hline
\end{tabular}

Tabelle 7: Durchschnittliche jährliche Genauigkeit von RW-Prognosen

Für jeden Prognosehorizont (in Jahren und nicht in Monaten, hier: Jahr $+n, n=1, \ldots, 5$ ) werden die mittleren relativen absoluten Fehler für alle Schweizer Aktien ermittelt und zu einem Durchschnitt pro Jahr aggregiert. Die Zeile „Gesamt“ beinhaltet den Durchschnitt der mittleren relativen absoluten Fehler über den Gesamtzeitraum pro Prognosehorizont. Die Sterne zeigen die Signifikanz der einseitigen t-Tests (Mittelwert von null) sofern die Werte in Tabelle 7 grösser sind als in Tabelle 6 während die Apostrophe die Signifikanz für Werte in Tabelle 7 die kleiner sind als in Tabelle 6 zeigen. $* / * 10 \%, * * / " 5 \%$, $* * * / “ 1 \%$. 
Die obigen Analysen zeigen, dass die Schätzungen der Analysten ungenau und tendenziell optimistisch verzerrt sind. Weiterhin ist eine pessimistische Verzerrung für kurzfristige Prognosehorizonte in den Jahren vor und direkt nach dem Höhepunkt der Finanzmarktkrise zu verzeichnen. Gleichzeitig sind die Schätzungen in den Krisenjahren relativ ungenau, was auf verzerrte und ungenaue Kapitalkosten hindeuten könnte. Allerdings ist gerade für kurzfristige Zeiträume - auch in den Krisenjahren - festzustellen, dass die einfache Fortschreibung des letzten Ergebnisses deutlich verzerrter und ungenauer ist als eine Prognose von Analysten. Dieses Ergebnis ist im Einklang mit der bestehenden Literatur, in der gezeigt wird, dass Analysten in der kurzen Frist relativ zu Zeitreihenprognosen einen zeitlichen Vorteil in der Verarbeitung von Informationen haben (Brown et al. 1987; Kross et al. 1990; Lys/Soo 1995) sowie einen Informationsvorteil besitzen (Fried/Givoly 1982).

Als Alternative zu den Analystenschätzungen wurde in jüngster Zeit verstärkt auf Korrekturen der Fehler in den Analystenschätzungen (Mohanram/Gode 2013) oder Querschnittsanalysen zur Vorhersage zukünftiger Unternehmensergebnisse auf der Basis von historischen Ergebnissen und Kennzahlen (Hou et al. 2012; Li/Mohanram 2014) zurückgegriffen. Für den US-amerikanischen Kapitalmarkt konnte bereits gezeigt werden, dass diese selbst generierten Prognosen zu Unternehmensergebnissen weniger verzerrt sind als Analystenschätzungen (Hou et al. 2012; Li/Mohanram 2014). Fraglich bleibt aber, ob diese vergangenheitsorientieren Prognosen auch in volatilen Zeiten zu genaueren Schätzern für künftige Ergebnisse führen. Betrachtet man die Resultate der naiven Extrapolation des letzten Unternehmensergebnisses so stellt man fest, dass Analystenprognosen gerade im kurzen Zeitraum schneller reagieren können. Dies stellt die Nützlichkeit von auf historischen Daten basierenden Vorhersagen von Unternehmensergebnissen zumindest in Frage. Eine weitere Problematik bei der Verwendung von historischen Prognosemodellen ist deren Rückgriff auf Daten des Rechnungswesens in der Regression, welche maximal quartalsweise zur Verfügung stehen. Für das hier verwendete Modell mit monatlicher (täglicher) Schätzung sind solche Ergebnisprognosen ohne unterjährige Aktualisierung nicht von Nutzen. Letztendlich führen solche vergangenheitsorientierten Ergebnisschätzungen auch dazu, dass der Schätzer für die impliziten Kapitalkosten nicht mehr zukunftsorientiert ist, da er mit vergangenen Daten operationalisiert wird.

Schlussendlich sollen die Ergebnisprognosen im Rahmen der impliziten Kapitalkostenermittlung aber möglichst genaue Schätzer für die aktuellen Markterwartungen darstellen. Es bleibt hier zumindest fraglich ob Marktteilnehmer sich - speziell in Krisenzeiträumen rein auf die mechanische Extrapolation historischer Zusammenhänge verlassen. Wenn die Erwartungen aller Marktteilnehmer ähnlich verzerrt und ungenau waren wie diejenigen der Analysten, dann sind die Prognosen der Analysten trotzdem ein guter Schätzer für die Markterwartungen und sollten nicht zu nach oben verzerrten Kapitalkosten geführt haben (Lewellen 2010).

Insgesamt bleibt jedoch das Problem bestehen, dass die Analystenschätzungen wohl zumindest zu einem gewissen Teil verzerrt sind und es ist mit bestehenden Methoden bislang nicht möglich die Abweichung der Analystenprognosen von den tatsächlichen Markterwartungen zu ermitteln. Ob die mit Hilfe von Querschnittsregressionen erstellten Ergebnisprognosen besser geeignet sind, die Markterwartungen - auch über die Zeit - abzubilden, bleibt zukünftiger Forschung vorbehalten. 


\section{Zusammenfassung}

In diesem Beitrag werden die Eigenkapitalkosten für Schweizer Unternehmen mit Hilfe von impliziten Kapitalkostenmodellen ermittelt. In einer Vielzahl von betriebswirtschaftlichen Fragestellungen werden Kapitalkosten mit Zukunftsbezug benötigt, welcher durch die Verwendung impliziter Kapitalkosten hergestellt werden kann. Die empirische Analyse des Schweizer Aktienmarktes zeigt, dass die aus den aktuellen Markterwartungen abgeleiteten Kapitalkosten mit sich verändernden konjunkturellen Gegebenheiten deutlichen Schwankungen unterliegen. So ist ein Anstieg der Marktrisikoprämie im Zuge der internationalen Finanzmarktkrise aber auch im Rahmen der zunehmenden Stärke des Schweizer Franken zu beobachten. Weiterhin zeigt sich, dass sich die unterschiedliche Sensitivität der Branchen auf makroökonomische Einflüsse auch in unterschiedlich stark ausgeprägten Veränderungen in den jeweiligen Risikoprämien auswirkt. Trotz dieser Vorteile der impliziten Kapitalkosten wird in diesem Beitrag auch auf die Problematik ungenauer und verzerrter Analystenschätzungen hingewiesen, die zu Schätzfehlern bei den Kapitalkosten führen können. Es wird gezeigt, dass die von Analysten erstellten Prognosen auch am Schweizer Aktienmarkt verzerrt sowie ungenau sind und somit keine gute Vorhersage für realisierte Gewinne darstellen. Obwohl Analystenprognosen trotzdem gute Schätzer für die Erwartungen aller Marktteilnehmer darstellen können, sollte sich der Nutzer von implizit ermittelten Kapitalkosten über potentielle Fehler in den Analystenschätzungen bewusst sein.

\section{Anhang}

Skalierung der relativen Fehler mit dem realisierten Ergebnis.

\begin{tabular}{|c|c|c|c|c|c|}
\hline Jahr & $\phi \mathbf{R F}_{\mathrm{Jahr}+1}$ & $\phi \mathbf{R F}_{\mathrm{Jahr}+2}$ & $\phi \mathrm{RF}_{\mathrm{Jahr}+3}$ & $\phi \mathbf{R F}_{\mathrm{Jahr}+4}$ & $\phi \mathbf{R F}_{\mathrm{Jahr}+5}$ \\
\hline 2006 & $0,06638^{* * *}$ & $0,10698 * * *$ & 0,00224 & $-0,13589 * * *$ & $-0,18841 * * *$ \\
\hline 2007 & $0,04283 * * *$ & $-0,10081 * * *$ & $-0,24398 * * *$ & $-0,27656 * * *$ & $-0,47667 * * *$ \\
\hline 2008 & $-0,08678 * * *$ & $-0,22487 * * *$ & $-0,22558 * * *$ & $-0,23774 * * *$ & $-0,32326^{* * *}$ \\
\hline 2009 & $-0,00189$ & $0,03092 * * *$ & 0,00081 & $-0,06049 * * *$ & $-0,10537^{* * *}$ \\
\hline 2010 & $-0,04932 * * *$ & $-0,09672 * * *$ & $-0,16808 * * *$ & $-0,16843 * * *$ & $-0,14554 * * *$ \\
\hline 2011 & $-0,10845 * * *$ & $-0,18455 * * *$ & $-0,23077 * * *$ & $-0,22553 * * *$ & \\
\hline 2012 & $-0,07960 * * *$ & $-0,12421 * * *$ & $-0,15687^{* * *}$ & & \\
\hline 2013 & $-0,04903 * * *$ & $-0,06497 * * *$ & & & \\
\hline 2014 & 0,00714 & & & & \\
\hline Gesamt & $-0,03048 * * *$ & $-0,07715 * * *$ & $-0,13957 * * *$ & $-0,16229 * * *$ & $-0,21108 * * *$ \\
\hline
\end{tabular}

Tabelle 8: Durchschnittliche jährliche Verzerrung der Analystenprognosen im Gesamtmarkt

Die Prognosefehler werden mit dem Absolutwert des realisierten Ergebnisses skaliert. Werte über 1 wurden aus der Stichprobe entfernt. Für jeden Prognosehorizont (in Jahren und nicht in Monaten, hier: Jahr + $\mathrm{n}, \mathrm{n}=1, \ldots, 5)$ werden die mittleren relativen Fehler für alle Schweizer Aktien ermittelt und zu einem Durchschnitt pro Jahr aggregiert. Die Zeile „Gesamt“ beinhaltet den Durchschnitt der mittleren relativen Fehler über den Gesamtzeitraum pro Prognosehorizont. Die Sterne zeigen die Signifikanz der einseitigen t-Tests (Mittelwert von null). * $10 \%, * * 5 \%, * * 1 \%$. 


\begin{tabular}{|c|c|c|c|c|c|}
\hline Jahr & QRAF $_{\mathbf{J a h r}+1}$ & $\phi \mathbf{R A F}_{\mathbf{J a h r}+2}$ & $\phi \mathbf{R A F} \mathbf{F}_{\mathbf{J a h r}+3}$ & $\boldsymbol{\phi R A F}_{\mathbf{J a h r}+4}$ & $\boldsymbol{\phi R A F}_{\mathbf{J a h r}+5}$ \\
\hline 2006 & $0,18007 * * *$ & $0,25695 * * *$ & $0,28242 * * *$ & $0,31060 * * *$ & $0,33077 * * *$ \\
\hline 2007 & $0,19506 * * *$ & $0,28030 * * *$ & $0,36303 * *$ & $0,37062 * * *$ & $0,52161 * * *$ \\
\hline 2008 & $0,22897 * * *$ & $0,31822 * * *$ & $0,32454 * * *$ & $0,38213 * * *$ & $0,44021 * * *$ \\
\hline 2009 & $0,21752 * * *$ & $0,31292 * * *$ & $0,32584 * * *$ & $0,34165 * * *$ & $0,33296 * * *$ \\
\hline 2010 & $0,23004 * * *$ & $0,32010 * * *$ & $0,33956 * * *$ & $0,33479 * * *$ & $0,36348 * * *$ \\
\hline 2011 & $0,26362 * * *$ & $0,32084 * * *$ & $0,33013 * * *$ & $0,35986 * * *$ & \\
\hline 2012 & $0,22633 * * *$ & $0,26051 * * *$ & $0,29587 * * *$ & & \\
\hline 2013 & $0,17688 * * *$ & $0,21095 * * *$ & & & \\
\hline 2014 & $0,12699 * * *$ & & & & \\
\hline Gesamt & $0,21303 * * *$ & $0,29209 * * *$ & $0,32486 * * *$ & $0,34471 * * *$ & $0,38042 * * *$ \\
\hline
\end{tabular}

Tabelle 9: Durchschnittliche jährliche Genauigkeit von Analystenprognosen

Die Prognosefehler werden mit dem Absolutwert des realisierten Ergebnisses skaliert. Werte über 1 wurden aus der Stichprobe entfernt. Für jeden Prognosehorizont (in Jahren und nicht in Monaten, hier: Jahr + $\mathrm{n}, \mathrm{n}=1, \ldots, 5)$ werden die mittleren relativen absoluten Fehler für alle Schweizer Aktien ermittelt und zu einem Durchschnitt pro Jahr aggregiert. Die Zeile „Gesamt“ beinhaltet den Durchschnitt der mittleren relativen absoluten Fehler über den Gesamtzeitraum pro Prognosehorizont. Die Sterne zeigen die Signifikanz der einseitigen t-Tests (Mittelwert von null). ${ }^{*} 10 \%, * 5 \%, * * * 1 \%$.

\section{Literaturhinweise}

Ballwieser, W. (2005): Die Ermittlung impliziter Eigenkapitalkosten aus Gewinnschätzungen und Aktienkursen: Ansatz und Probleme, in: Schneider, D. et al. (Hrsg.): Kritisches zu Rechnungslegung und Unternehmensbesteuerung. Festschrift zur Vollendung des 65. Lebensjahres von Theodor Siegel, Berlin, S. 321-337.

Bassemir, M./Gebhardt, G./Ruffing, P. (2012): Zur Diskussion um die (Nicht-)Berücksichtigung der Finanz- und Schuldenkrisen bei der Ermittlung der Kapitalkosten, in: Die Wirtschaftsprüfung, Jg. 65, Nr. 16, S. 882-892.

Berndt, T./Froese, H./Leverkus, L./Ornik, R. (2014): Comply or Explain als Lösung für Schweizer Fairness Opinions? - Bewertungsunterschiede und Intransparenz analysiert - Verbesserungen identifiziert, in: Der Schweizer Treuhänder, Nr. 9/2014, S. 748-753.

Bessler, W./Stanzel, M. (2007): Qualität und Effizienz der Gewinnprognosen von Analysten: Eine empirische Untersuchung für den deutschen Kapitalmarkt, in: Kredit und Kapital, Jg. 40, Nr. 1, S. 89-129.

Bolliger, G. (2004): The characteristics of individual analysts' forecasts in Europe, in: Journal of Banking and Finance, Vol. 28, No. 9, S. 2283-2309.

Botosan, C. A./Plumlee, M. A. (2005): Assessing Alternative Proxies for the Expected Risk Premium, in: The Accounting Review, Vol. 80, No. 1, S. 21-53.

Bradshaw, M. T./Drake, M. S./Myers, J. N./Myers, L. A. (2012): A re-examination of analysts' superiority over time-series forecasts of annual earnings, in: Review of Accounting Studies, Vol. 17, No. 4, S. 944-968.

Brown, L. D./Hagerman, R. L./Griffin, P. A./Zmijewski, M. E. (1987): Security analyst superiority relative to univariate time-series models in forecasting quarterly earnings, in: Journal of Accounting and Economics, Vol. 9, No. 1, S. 61-87. 
Brown, L. D. (1996): Analyst Forecasting Errors and Their Implications for Security Analysis: An Alternative Perspective, in: Financial Analysts Journal, Vol. 52, No. 1, S. 40-47.

Capstaff, J./Paudyal, K./Rees, W. (1998): Analysts’ Forecasts of German Firms' Earnings: a Comparative Analysis, in: Journal of International Financial Management and Accounting, Vol. 9, No. 2, S. 83-116.

Capstaff, J./Paudyal, K./Rees, W. (2001): A Comparative Analysis of Earnings Forecasts in Europe, in: Journal of Business Finance \& Accounting, Vol. 28, No. 5, S. 531-562.

Claus, J./Thomas, J. (2001): Equity Premia as Low as Three Percent? Evidence from Analysts' Earnings Forecasts for Domestic and International Stock Markets, in: The Journal of Finance, Vol. 56, No. 5, S. 1629-1666.

Daske, H./Gebhardt, G. (2006): Zukunftsorientierte Bestimmung von Risikoprämien und Eigenkapitalkosten für die Unternehmensbewertung, in: Zeitschrift für betriebswirtschaftliche Forschung und Praxis, Jg. 58, Nr. 4, S. 530-551.

Daske, H./Gebhardt, G./Klein, S. (2006): Estimating the Expected Cost of Equity Capital Using Analysts' Consensus Forecasts, in: schmalenbach business review, Vol. 58, No. 1, S. 2-36.

Easterwood, J. C./Nutt, S. R. (1999): Inefficiency in Analysts’ Earnings Forecasts: Systematic Misreaction or Systematic Optimism?, in: The Journal of Finance, Vol. 54, No. 5, S. 1777-1797.

Easton, P./Taylor, G./Shroff, P./Sougiannis, T. (2002): Using Forecasts of Earnings to Simultaneously Estimate Growth and the Rate of Return on Equity Investment, in: Journal of Accounting Research, Vol. 40, No. 3, S. 657-676.

Easton, P.D./Monahan, S. J. (2005): An Evaluation of Accounting-Based Measures of Expected Returns, in: The Accounting Review, Vol. 80, No. 2, S. 501-538.

Easton, P. D./Sommers, G. A. (2007): Effect of Analysts' Optimism on Estimates of the Expected Rate of Return Implied by Earnings Forecasts, in: Journal of Accounting Research, Vol. 45, No. 5, S. 983-1015.

Easton, P. (2009): Estimating the Cost of Capital Implied by Market Prices and Accounting Data, in: Foundations and Trends ${ }^{\circledR}$ in Accounting, Vol. 2, No. 4, S. 241-364.

Economiesuisse (2011a): Wachstumsdynamik schwächt sich ab, Medienmitteilung vom 9.6.11, abrufbar unter: http://www.economiesuisse.ch/de/PDF\%20Download\%20Files/MM\%20Konjunkt urprognose\%20_20110609.pdf (16.1.2015, 14 Uhr).

Economiesuisse (2011b): 2012: Tiefes Wachstum in einem Jahr der Transformation, Medienmitteilung vom 5.12.11, abrufbar unter: http://www.economiesuisse.ch/de/PDF\%20Download\%20Fil es/MM_Konjunktur_2012_D.pdf (16.1.2015, 14 Uhr).

Fama, E. F./French, K. R. (1997): Industry costs of equity, in: Journal of Financial Economics, Vol. 43, No. 2, S. 153-193.

Fama, E. F./French, K. R. (2000): Forecasting Profitability and Earnings, in: The Journal of Business, Vol. 73, No. 2, S. 161-175.

Fama, E. F./French, K. R. (2006): Profitability, investment and average returns, in: Journal of Financial Economics, Vol. 82, No. 3, S. 491-518.

Feltham, G. A./Oblson, J. A. (1995): Valuation and Clean Surplus Accounting for Operating and Financial Activities, in: Contemporary Accounting Research, Vol. 11, No. 2, S. 689-731.

Feltham, G. A./Oblson, J. A. (1996): Uncertainty Resolution and the Theory of Depreciation Measurement, in: Journal of Accounting Research, Vol. 34, No. 2, S. 209-234.

Fried, D./Givoly, D. (1982): Financial analysts' forecasts of earnings: A better surrogate for market expectations, in: Journal of Accounting and Economics, Vol. 4, No. 2, S. 85-107. 
Gebhardt, W. R./Lee, C. M. C./Swaminathan, B. (2001): Toward an Implied Cost of Capital, in: Journal of Accounting Research, Vol. 39, No. 1, S. 135-176.

Gebhardt, G./Mansch, H. (2005): Wertorientierte Unternehmenssteuerung in Theorie und Praxis, in: Zeitschrift für betriebswirtschaftliche Forschung und Praxis, Sonderheft 53, S. 1-192.

Hou, K./Robinson, D. T. (2006): Industry Concentration and Average Stock Returns, in: The Journal of Finance, Vol. 61, No. 4, S. 1927-1956.

Hou, K./van Dijk, M. A./Zhang, Y. (2012): The implied cost of capital: A new approach, in: Journal of Accounting and Economics, Vol. 53, No. 3, S. 504-526.

Hou, K./van Dijk, M. A. (2014): Resurrecting the Size Effect: Firm Size, Profitability Shocks and Expected Stock Returns, Working Paper, abrufbar unter: http://ssrn.com/abstract=1536804 (16.1.2015, $15 \mathrm{Uhr}$ ).

Kroos, W./Ro, B./Schroeder, D. (1990): Earnings Expectations: The Analysts' Information Advantage, in: The Accounting Review, Vol. 65, No. 2, S. 461-476.

Lee, C. M. C./So, E. C./Wang, C. C. Y. (2014): Evaluating Firm-Level Expected-Return Proxies, Working Paper, abrufbar unter: http://ssrn.com/abstract=1653940 (15.1.2015, 10 Uhr).

Lewellen, J. (2010): Accounting anomalies and fundamental analysis: An alternative view, in: Journal of Accounting and Economics, Vol. 50, No. 2-3, S. 455-466.

Li, K. K. (2011): How well do investors understand loss persistence?, in: Review of Accounting Studies, Vol. 16, No. 3, S. 630-667.

Li, K. K./Mohanram, P. (2014): Evaluating cross-sectional forecasting models for implied cost of capital, in: Review of Accounting Studies, Jg. 19, Nr. 3, S. 1152-1185.

Loderer, C./Jörg, P./Pichler, K./Roth, L./Wälchli, U./Zgraggen, P. (2010): Handbuch der Bewertung Band 1: Projekte, 5. Aufl., Zürich.

Lücke, W. (1955): Investitionsrechnung auf der Grundlage von Ausgaben oder Kosten?, in: Zeitschrift für betriebswirtschaftliche Forschung und Praxis, Jg. 7, S. 310-324.

Lys, T./Soo, L.G. (1995): Analysts' Forecast Precision as a Response to Competition, in: Journal of Accounting, Auditing and Finance, Vol. 10, No. 4, S. 751-765.

Mohanram, P./Gode, D. (2013): Removing predictable analyst forecast errors to improve implied cost of equity estimates, in: Review of Accounting Studies, Vol. 18, No. 2, S. 443-478.

Ohlson, J.A. (1995): Earnings, Book Values, and Dividends in Equity Valuation, in: Contemporary Accounting Research, Vol. 11, No. 2, S. 661-687.

Pictet \& Cie SA (2015): Die Performance von Aktien und Obligationen in der Schweiz (1926-2014), Januar 2015, abrufbar unter: http://www.pictet.com/corporate/de/home/media_relations/corpora te_publications/special/performance.html (6.7.2015, $11 \mathrm{Uhr})$.

Preinreich, G. A. D. (1937): Valuation and Amortization, in: The Accounting Review, Vol. 12, No. 3, S. 209-226.

Schneller, T./Schwendener, P./Elsaesser, P. (2010): Risiken von Aktienanlagen - Bestimmung der Marktrisikoprämie, in: Der Schweizer Treuhänder, Nr. 10/2010, S. 658-665.

Stewart, G. B. (1991): The Quest for Value - A Guide for Senior Managers, New York.

Volkart, R. (2010): Unternehmensbewertung und Akquisitionen, 3. Aufl., Zürich.

Volkart, R./Vettiger, T./Forrer, F. (2013): Bestimmung der Kapitalkosten im Rahmen der finanziellen Führung: klassische und neue Herausforderungen aus nationaler und internationaler Sicht, in: Seicht, G. (Hrsg.): Jahrbuch für Controlling und Rechnungswesen 2013, Wien, S. 101-126. 
Wallmeier, M. (2005): Gewinnprognosen von Finanzanalysten: Ein europäischer Vergleich, in: Finanz Betrieb, Jg. 7, Nr. 11, S. 744-750.

Wallmeier, M. (2007): Implizite Kapitalkostensätze und der Fortführungswert im Residualgewinnmodell, in: Betriebswirtschaftliche Forschung und Praxis, Jg. 59, Nr. 6, S. 558-579.

Wallmeier, M. (2008): Der „Steady State“: Das Phantom der Unternehmensbewertung, in: Laitenberger, J./Löffler, A. (Hrsg.): Finanzierungstheorie auf vollkommenen und unvollkommenen Kapitalmärkten. Festschrift für Lutz Kruschwitz zum 65. Geburtstag, München, S. 139-156.

Patricia Ruffing-Straube, Dr., ist Postdoktorierende am Institut für Betriebswirtschaftslehre an der Universität Zürich, Lehrstuhl für Accounting, insbesondere Unternehmensrechnung und Controlling.

Anschrift: Universität Zürich, Institut für Betriebswirtschaftslehre, Lehrstuhl für Accounting, insbes. Unternehmensrechnung und Controlling, Affolternstrasse 56, CH-8050 Zürich, Tel.: +41 (0) 4463429 82, Fax: +41 (0) 4463449 12, E-Mail: patricia.ruffing@business.uzh.ch. 